\title{
Two new ZZ Ceti pulsators from the HS and HE surveys ${ }^{\star}$
}

\author{
R. Silvotti ${ }^{1}$, B. Voss ${ }^{2}$, I. Bruni ${ }^{3}$, D. Koester ${ }^{2}$, D. Reimers ${ }^{4}$, R. Napiwotzki ${ }^{5}$, and D. Homeier ${ }^{6}$ \\ 1 INAF - Osservatorio Astronomico di Capodimonte, via Moiariello 16, 80131 Napoli, Italy \\ e-mail: silvotti@na.astro.it \\ 2 Institut für Theoretische Physik und Astrophysik der Universität Kiel, Leibnizstraße 15, 24098 Kiel, Germany \\ e-mail: voss@astrophysik.uni-kiel.de,koester@astrophysik.uni-kiel.de \\ 3 INAF - Osservatorio Astronomico di Bologna, via Ranzani 1, 40127 Bologna, Italy \\ e-mail: ivan.bruni@bo.astro.it \\ 4 Hamburger Sternwarte, Gojenbergsweg 112, 21029 Hamburg, Germany \\ e-mail: dreimers@hs.uni-hamburg.de \\ 5 Centre for Astrophysics Research, University of Hertfordshire, College Lane, Hatfield AL10 9AB, UK \\ e-mail: r.napiwotzki@star.herts.ac.uk \\ ${ }^{6}$ Institut für Astrophysik, Georg-August-Universität, Friedrich-Hund-Platz 1, 37077 Göttingen, Germany \\ e-mail: derek@astro.physik.uni-goettingen.de
}

Received 3 May 2005 / Accepted 9 June 2005

\section{ABSTRACT}

We report the detection of nonradial $g$-mode oscillations in the DA white dwarfs HS 1039+4112 $(B=15.9)$ and HE $1429-0343(B=15.8)$ from time-series photometry made at the Loiano $1.5 \mathrm{~m}$ telescope. The two stars were previously selected as probable pulsators based on two-color photometry and spectral analysis respectively. Following our temperature and surface gravity determinations, HS $1039+4112\left(T_{\text {eff }}=11200 \mathrm{~K}\right.$, $\log g=8.2)$ is located near the red edge of the ZZ Ceti instability strip, whereas HE $1429-0343\left(T_{\text {eff }}=11400 \mathrm{~K}\right.$, $\left.\log g=7.8\right)$ falls in the middle of the strip. Both stars show a multi-mode behavior with the main periods at about 850 and 970 s respectively, and relatively large amplitudes $(\sim 7 \%$ and $\sim 2.5 \%)$.

Key words. stars: white dwarfs - stars: oscillations - stars: individual: HS 1039+4112, HE 1429-0343

\section{Introduction}

The ZZ Ceti stars (or DAVs=DA Variables) are old DA (H-rich) white dwarfs (WDs) showing multi-periodic luminosity variations with typical periods between $\sim 100$ and $\sim 1200$ s and amplitudes between a few percent down to less than $0.1 \%$, generally interpreted as nonradial $g$-mode oscillations.

The importance of studying the ZZ Ceti variables is related to the possibility, given by seismological analysis, of studying in detail their interiors and deriving numerous basic stellar parameters such as the mass, the rotation and the thickness of the external layers of $\mathrm{H}$ and $\mathrm{He}$. Due to the high stability of the stellar structure, the pulsation periods of the DAV white dwarfs are extremely stable in time, with values of $\dot{P}$ that can be as small as $\approx 10^{-15}$ (Mukadam et al. 2003, and references therein). More in general, WD seismology can help to improve our knowledge of the poorly known rate of the ${ }^{12} \mathrm{C}(\alpha, \gamma){ }^{16} \mathrm{O}$ nuclear reaction (see e.g. Metcalfe 2003 and Straniero et al. 2003 and references

\footnotetext{
* Based on observations collected at the Loiano Observatory, operated by the Istituto Nazionale di Astrofisica (INAF), and at the Centro Astronomico Hispano Aleman (CAHA) at Calar Alto, operated jointly by the Max-Planck Institut für Astronomie and the Instituto de Astrofisica de Andalucia (CSIC). Partially based on data obtained at the Paranal Observatory of the European Southern Observatory for programmes 165.H-0588 and 167.D-0407.
}

therein), and of the WD cooling rates, including crystallization processes (Kanaan et al. 2005, and references therein; see also Fontaine et al. 2001 for a detailed review on this argument).

The effective temperatures of the ZZ Ceti stars are confined between about 10700 and $12100 \mathrm{~K}$ for a canonical surface gravity $\log g \sim 8.0$ (Bergeron et al. 1995, 2004; Koester \& Holberg 2001; Mukadam et al. 2004a). The blue edge of the DAV instability strip can be interpreted as the limit where the base of the surface convective zone becomes sufficiently deep for the local thermal time-scale to be comparable to the shortest observable $g$-mode pulsation period $(\approx 100$ s for typical WD masses, see Tassoul et al. 1990, and references therein).

This scenario is in agreement with an observed trend on temperature: the hottest DAVs have shorter pulsation periods and lower amplitudes than the cooler ones. More uncertain is the interpretation of the red edge: convection-pulsation interaction (Winget \& Fontaine 1982), convective mixing between hydrogen and helium (Bergeron et al. 1990) or another mechanism? This is related to the driving of the pulsations which could be based on the $\kappa-\gamma$ mechanism, active in a hydrogen layer near the surface (Dziembowski \& Koester 1981; Dolez \& Vauclair 1981; Winget et al. 1982), although an alternative "convective driving" has been proposed by Brickhill (1991, see also Gautschy et al. 1996; and Goldreich \& Wu 1999). 
Recently, thanks to the results of the Sloan Digital Sky Survey, the number of known ZZ Ceti pulsators has increased by a factor of two, reaching a total number of 83 (Bergeron et al. 2004; Mukadam et al. 2004a; Mullally et al. 2005; Silvotti et al. in preparation ${ }^{1}$ ). Moreover another 5 (possibly 6) DAVs have been observed in cataclysmic variable systems (Woudt \& Warner 2004; Warner \& Woudt 2005; Araujo-Betancor et al. 2005). Following Mukadam et al. (2004b), it is still not clear whether there might be some non-variable stars within the strip, although these stars could simply have very low pulsation amplitudes, below the detection limit.

In this article we present two new ZZ Ceti stars, selected from the HS (Hamburg Schmidt, Hagen et al. 1995) and HE (Hamburg ESO, Christlieb et al. 2001) surveys, which bring the total number of known non-interacting ZZ Ceti to 85 .

\section{Selection of the $Z Z$ Ceti candidates}

The candidates were selected based on two-color photometry and spectral analysis.

\subsection{Two-color Strömgren photometry}

A list of 3000 possible cool white dwarf stars was derived from the low-resolution photographic objective prism spectra of the Hamburg Quasar Survey (HQS) by Homeier \& Koester (2001). One thousand of these objects are cool ( $\left.T_{\text {eff }} \lesssim 16000 \mathrm{~K}\right)$ and, because of the large errors on the temperature (up to $\sim 6000 \mathrm{~K}$ ), all of them are potential ZZ Ceti pulsators. In order to select the best ZZ Ceti candidates, preparatory observations are currently being conducted, aimed to derive more precise temperatures and gravities for the $\approx 500$ brightest stars $(B<16.5 \mathrm{mag}$ ).

We obtained photometry in the Strömgren $u, b, y$ and Johnson $I$ bands at the Calar Alto $2.2 \mathrm{~m}$ telescope using the BUSCA four-channel CCD camera (Reif et al. 1999) ${ }^{2}$. The colours $u-b$ and $b-y$ show a strong dependency on $T_{\text {eff }}$ and $\log g$, which is strongest in the region of the ZZ Ceti instability strip. In the course of our analysis, we noticed technical problems with the BUSCA $y$ filter as well as an even stronger temperature dependency of $b-I$ than that of $b-y$. Therefore we use the $u-b$ vs. $b-I$ two-color diagram for the analysis of our data.

To derive atmospheric parameters from the two-color data, we compare them to a grid of synthetic colors. This grid was computed from Koester DA model atmospheres (described e.g. in Finley et al. 1997) by convolving the model spectra with the BUSCA filter transmission curves. Temperatures and gravities were determined by interpolating linearly between the grid points. The observational and calibration errors of the colors are $\sim 0.02 \mathrm{mag}$, which translates into an error of $\sim \pm 0.1$ in $\log g$ and $\sim \pm 300 \mathrm{~K}$ in $T_{\text {eff }}$. This temperature error is small compared to the width of the instability strip, and thus most objects are clearly placed either outside or inside the instability strip by the photometry.

\footnotetext{
${ }^{1}$ Nonradial oscillations were recently detected in GD 133 from time-series photometry performed in May 2005.

${ }^{2}$ See also http://www. caha.es/guijarro/BUSCA/intro.html
}

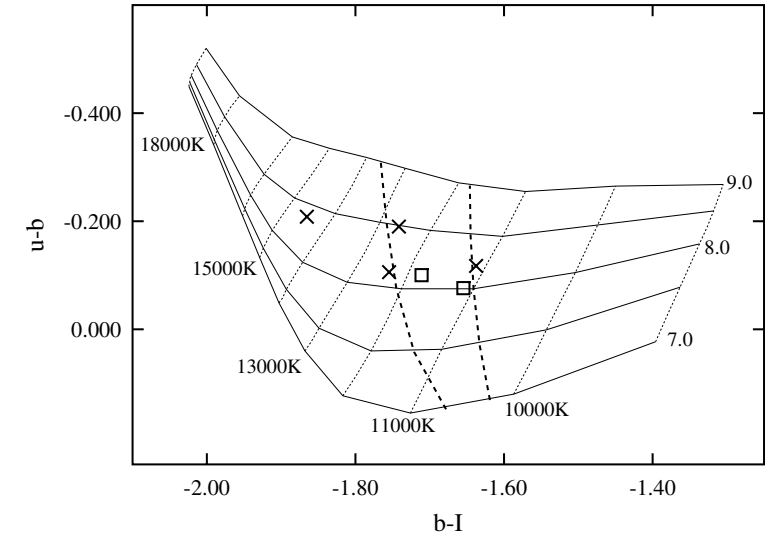

Fig. 1. Two-color data and the grid of synthetic colors from model atmospheres. Solid lines are lines of constant gravity and dotted lines are lines of constant temperature $\left(T_{\mathrm{eff}}=20000 \mathrm{~K}, 18000,16000\right.$, 15000 and down to 9000 in steps of $1000 \mathrm{~K})$. Dashed lines show the boundaries of the instability strip, according to Bergeron et al. (2004). The crosses represent the four stars selected as possible pulsators: from left to right HS 1610+1639, HS 1443+2934, HS 1253+1033 and HS 1039+4112. The squares are two previously known ZZ Ceti stars, GD 244 (left) and PG 2303+242, that were included in the preparatory observations as a check of our methods.

Four objects that were selected as potential pulsators in this way are studied here. Their two-color data are plotted in Fig. 1, together with the temperature/gravity grid and the instability strip edges, taken from Bergeron et al. (2004). For one of the new pulsators, HS 1039+4112 $\left(\mathrm{RA}_{2000}=104233.5\right.$, $\left.\mathrm{Dec}_{2000}=+405716, B=15.9 \mathrm{mag}\right)$, we find $T_{\text {eff }}=11200 \pm$ $270 \mathrm{~K}$ and $\log g=8.2 \pm 0.12$. The parameters of the three remaining objects are given in Table 2 .

The selection of the object HS $1610+1639$ is problematic: using the now obsolete $b-y$ data, we obtained $T_{\text {eff }}=11700 \mathrm{~K}$ and $\log g=8.2$, corresponding to the middle of the instability strip. This is the reason why HS $1610+1639$ was included in the sample observed at Loiano. However, after the time series observations at Loiano had been made, the re-analysis of the data using the $b-I$ color yielded a higher temperature for this object, $T_{\text {eff }}=14500 \mathrm{~K}$ (and $\log g=8.4$ ), definitively too hot for ZZ Ceti pulsations.

\subsection{Spectral analysis}

The other new pulsator, HE 1429-0343 $\left(\mathrm{RA}_{2000}=1432\right.$ 03.2, Dec $_{2000}=-035638, B=15.8$ ), was selected based on high-resolution spectra from the SPY survey (Napiwotzki et al. 2003). An analysis of these spectra for 754 white dwarfs, many of which are first spectroscopic confirmations of WDs from the HS and HE surveys, was conducted by Voss et al. (2005, in preparation). Details about theoretical spectra and fitting procedures can be found in Finley et al. (1997). Figure 2 displays the model atmosphere fit for HE 1429-0343, from which we obtain $T_{\text {eff }}=11434 \pm 36 \mathrm{~K}$ and $\log g=7.82 \pm 0.02$.

The other object selected from spectroscopy is HS $1641+7132$, one of the 12 possible DAVs identified from medium-resolution spectroscopy by Homeier et al. (1998). HS 1641+7132 was selected for time-series photometry 


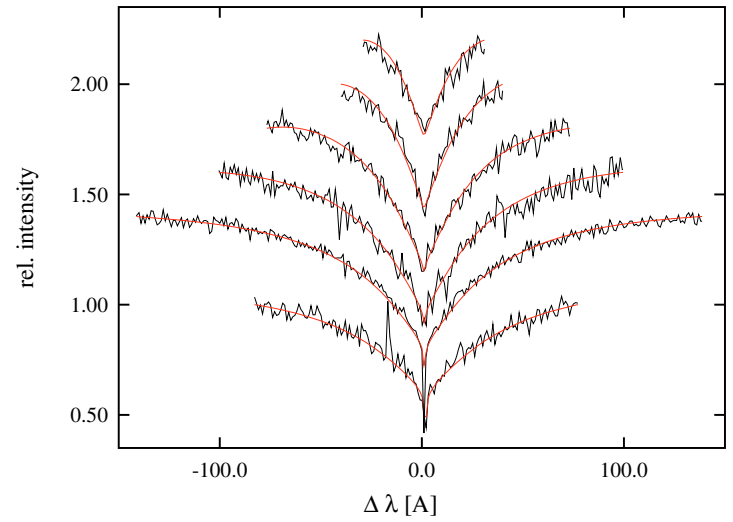

Fig. 2. The SPY spectrum and fit of HE 1429-0343. The spectrum was rebinned to a resolution of $1 \AA$ for this plot, the fit was done with the original resolution of $0.1 \AA$. The deep core of $\mathrm{H} \beta$ is not real but is caused by a cosmic ray, which however does not significantly affect the fitting process.

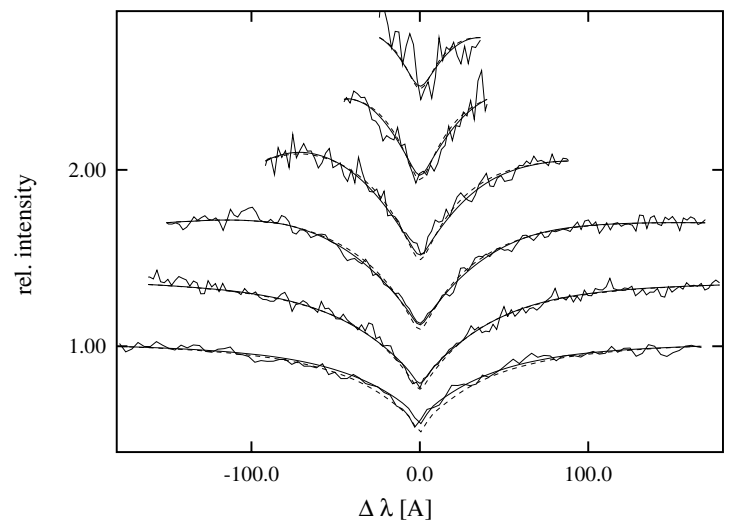

Fig. 3. Spectrum of HS $1641+7132$ and fits for $T_{\text {eff }}=15350 \mathrm{~K}$, $\log g=7.9$ (solid line), and for $11800 \mathrm{~K}, \log g=8.1$ (dashed)

because of its atmospheric parameters, $T_{\text {eff }}=11600 \pm 80 \mathrm{~K}$ and $\log g=8.0 \pm 0.06$, which correspond to the middle of the instability strip. While the formal errors are small despite the low $S / N$ and the resolution $(6 \AA)$, line profile fits near the Balmer line maximum are known to easily lead to degenerate solutions for $T_{\text {eff }}$ and $\log g$. Indeed, upon re-analyzing the original data from 1998, we found that even small changes to the startup values of the fitting procedure would produce temperatures as much as $200 \mathrm{~K}$ apart, with a mean value of $11800 \mathrm{~K}$. More important, we found a second solution for the fit at a much higher temperature $T_{\text {eff }}=15350 \pm 300 \mathrm{~K}$ and $\log g=7.88 \pm 0.06$, with a reduced $\chi 2=1.26$ as compared to 1.45 for the cooler solution. The two fits of the spectrum are shown in Fig. 3.

\section{Time-series photometry}

The time-series observations were performed in June 2004 at the $1.5 \mathrm{~m}$ Loiano telescope using the $\mathrm{BFOSC}^{3}$ instrument without any filter. A recent version of the software allows one to launch a series of exposures where the CCD is read only in a

${ }^{3}$ Bologna Faint Object Spectrograph \& Camera, see http://www.bo.astro.it/loiano/ for more details.
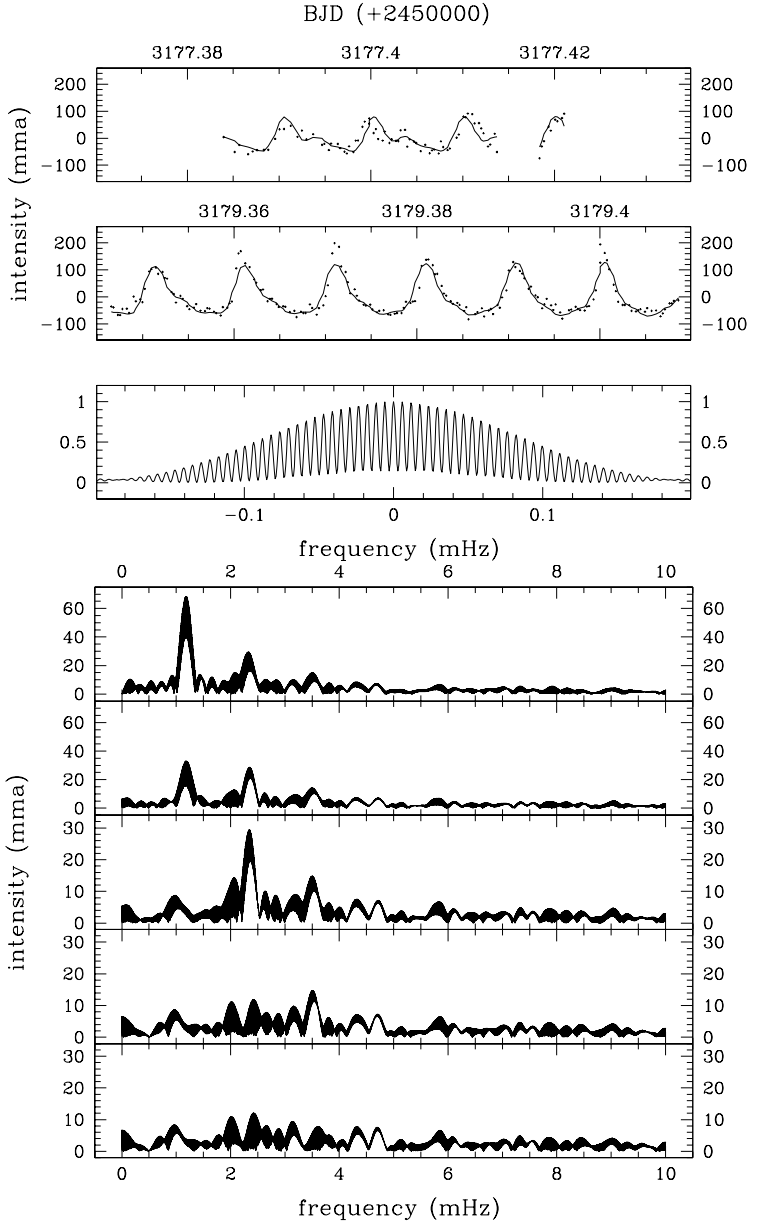

Fig. 4. Light curves, spectral window and amplitude spectrum of HS $1039+4112(1 \mathrm{mma}=0.1 \%$ change in intensity). The observations were performed on June 20 and 22, 2004. The amplitude spectrum is obtained joining together the two runs in order to increase the frequency resolution. The lower panels show the behavior of the spectrum when we subtract, at each iteration, the highest peak (prewhitening); note that the intensity scale is not the same in all panels.

small box around the target, in order to decrease the read-out time. With a box of typically $\sim 300 \times 400$ pixels, we were able to measure at least two reference stars in each field. The integration times used were between 12 and $18 \mathrm{~s}$, giving an effective resolution of about 24 to $30 \mathrm{~s}$, because of the CCD reading plus some extra time due to internal control of the software.

The data were then reduced using $\mathrm{IRAF}^{4}$ aperture photometry. The flux of each target was divided by a combination of the reference stars with different weights, and then normalized to the mean ratio. Finally a residual extinction correction was applied and the times were converted to Barycentric Julian Date (BJD), using the algorithm of Stumpff (1980).

The light curves of the two new ZZ Ceti stars are shown in Figs. 4 and 5. The variable amplitudes in the light curves, presumably due to beating effects, suggest that both targets have

\footnotetext{
${ }^{4}$ Image Reduction and Analysis Facility, written and supported by the IRAF programming group at the National Optical Astronomy Observatories (NOAO) in Tucson, Arizona (http://iraf.noao.edu/).
} 

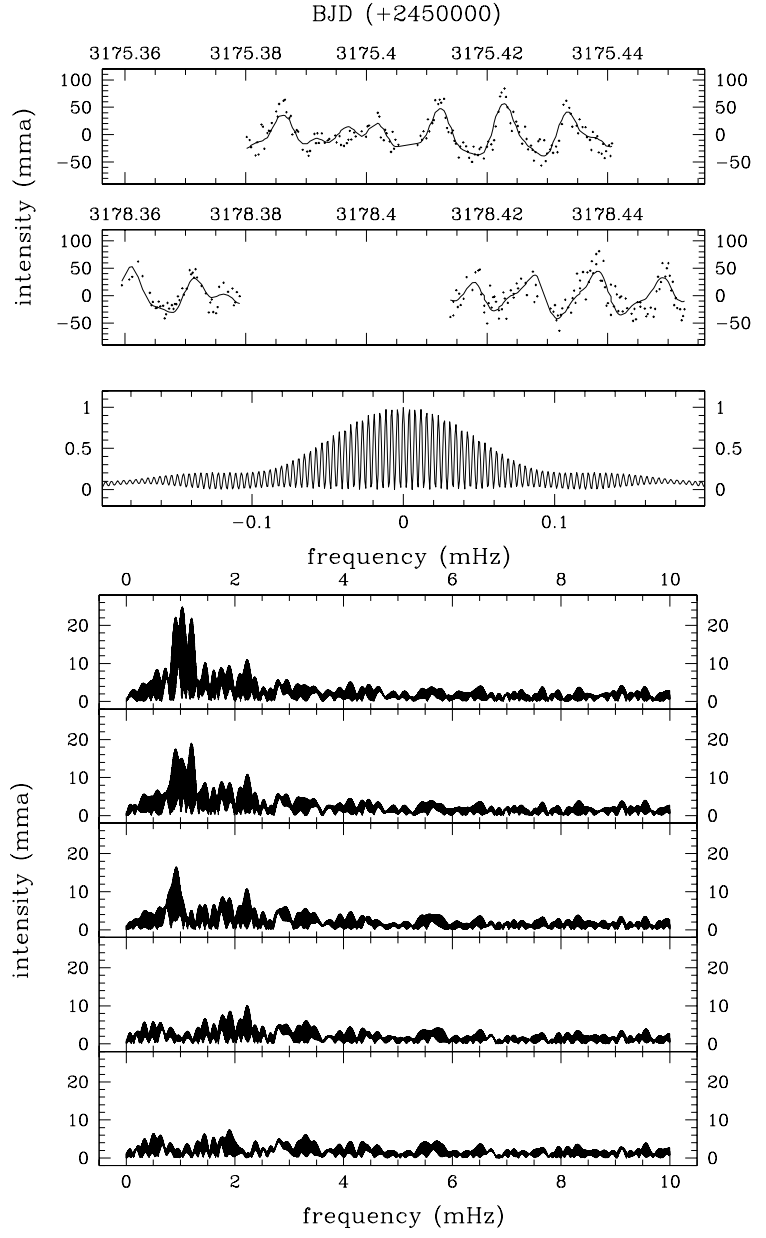

Fig. 5. Same as Fig. 4, but for HE 1429-0343. The observations of HE 1429-0343 were performed on June 18 and 21, 2004.

more than one excited pulsation mode. Each target was observed on two different nights to confirm the pulsations. In this way, joining together the two runs, the frequency resolution of the Fourier Transform is improved and it is possible to make a first separation of the different peaks (Figs. 4 and 5). The light curve of HS 1039+4112 has the typical large amplitude of many of the cool ZZ Ceti; moreover the spectrum shows the possible presence of the first and second harmonic of the main peak at $1.181 \mathrm{mHz}(846.6 \mathrm{~s})$. In the case of HE 1429-0343, which is located more close to the center of the instability strip, the pulsation amplitude is lower. Close to the highest peak of the spectrum at $1.032 \mathrm{mHz}(969.0 \mathrm{~s})^{5}$ there are at least two other signals at 0.922 and $1.206 \mathrm{mHz}(1084.9$ and $829.3 \mathrm{~s}$ respectively).

In Table 1 we report an attempt to fit the observed light curves (both nights together) with sinusoidal fits; the results of the fits are also shown in the upper panels of Figs. 4 and 5. However, due to the poor coverage, the pulsation spectra are not resolved and therefore these results should be taken with some prudence, in particular regarding the frequencies with lower amplitudes (3F1 and F4), which are quite close to the noise level. Concerning HS 1039+4112, the first and second

\footnotetext{
5 The amplitude of this mode, however, is not the highest following the results of the sinusoidal fits, as reported in Table 1.
}

Table 1. Results of the non-linear least-squares fits.

\begin{tabular}{lcccc}
\hline \hline Name & & $F(\mathrm{mHz})$ & $P(\mathrm{~s})$ & $A(\mathrm{mma})$ \\
\hline HS 1039+4112 & F1 & 1.169 & 855.5 & 55.2 \\
& & {$[1.181]$} & {$[846.6]$} & {$[71.8]$} \\
& F2 & 1.194 & 837.3 & 26.0 \\
& & & & {$[30.7]$} \\
& $2 \mathrm{~F} 1$ & 2.338 & 427.8 & 29.1 \\
& 3F1 & 3.507 & 285.1 & 14.6 \\
\hline HE 1429-0343 & F1 & 0.922 & 1084.9 & 16.3 \\
& F2 & 1.032 & 969.0 & 12.7 \\
& F3 & 1.206 & 829.3 & 18.3 \\
& F4 & 2.222 & 450.1 & 10.2 \\
\hline
\end{tabular}

harmonics suggest that the true value of the main frequency could be $1.169 \mathrm{mHz}$, whereas the value of 1.181 detected in the power spectrum could correspond to the 1-day alias $(1.169+$ $0.012 \mathrm{mHz}$ ). Indeed, using $1.169 \mathrm{mHz}$, the least-squares fit is more stable and therefore we adopt this solution in Table 1 (the alternative solution is also given in square brackets for completeness). In the case of HE 1429-0343, the results of the sinusoidal fits favor a higher amplitude of $\mathrm{F} 3$ and $\mathrm{F} 1$ respect to F2 which, however, is the highest peak in the Fourier spectrum (24.9 mma).

\section{The ZZ Ceti instability strip updated}

In Fig. 6 the location of the two new ZZ Ceti stars in a $\left(T_{\text {eff }}\right.$, $\log g$ ) plane is shown and compared with the edges of the observational ZZ Ceti instability strip as defined by Bergeron et al. (2004). Both stars are within the strip, although HS 1039+4112 is very close to the red edge.

On the other hand, if we consider the other four targets observed in the same run at Loiano, none of them shows pulsations. Their upper limits (i.e. the amplitude of the highest peak in the region of interest of their temporal spectra) are reported in Table 2, together with their B magnitudes, effective temperatures and surface gravities, date and duration of the observations.

Concerning HS $1641+7132$, its non-variability is compatible with the higher temperature solution which was found upon re-analyzing the spectroscopic data, as described in Sect. 2.2. Therefore we conclude that this star is likely to be a nonvariable DA with $T_{\text {eff }}$ between 15000 and $16000 \mathrm{~K}$. The same applies to HS $1610+1639$, which is clearly far from the blue edge of the instability strip, having an effective temperature of about $14500 \mathrm{~K}$.

Finally, concerning the other two stars that do not show luminosity variations, HS $1253+1033$ and HS $1443+2934$, both lie near the blue edge. The case of HS $1253+1033$ is the most intriguing as this star apparently falls inside the observational strip of Bergeron et al. (2004). However, even in this case, the relatively large uncertainties in $T_{\text {eff }}$ and $\log g$ cannot completely exclude the possibility that HS $1253+1033$ is actually slightly beyond the blue edge. Moreover, looking at Fig. 6, both stars would be outside the observational strip as defined 
Table 2. Objects which do not show pulsations.

\begin{tabular}{lcccccccc}
\hline \hline Name & $\mathrm{RA}_{2000}$ & Dec $_{2000}$ & $B$ & $\begin{array}{c}T_{\text {eff }} \\
(\mathrm{K})\end{array}$ & $\begin{array}{c}\log g \\
(\mathrm{cgs})\end{array}$ & $\begin{array}{c}\text { UT date } \\
\text { at start }\end{array}$ & $\begin{array}{c}D \\
(\mathrm{~h})\end{array}$ & $\begin{array}{c}L \\
(\mathrm{mma})\end{array}$ \\
\hline HS 1253+1033 & 125628.5 & +101709 & 14.4 & 12600 & 8.5 & $16 / 06 / 04$ & 1.6 & 5.3 \\
HS 1443+2934 & 144528.5 & +292131 & 14.5 & 12400 & 8.1 & $22 / 06 / 04$ & 2.0 & 1.5 \\
& & & & & & $23 / 06 / 04$ & 1.5 & 1.2 \\
HS 1610+1639 & 161302.4 & +163156 & 15.8 & 14500 & 8.4 & $17 / 06 / 04$ & 3.4 & 2.0 \\
HS 1641+7132 & 164100.0 & +712659 & 16.7 & $15300^{*}$ & $7.9^{*}$ & $23 / 06 / 04$ & 1.4 & 4.6 \\
\hline
\end{tabular}

${ }^{*}$ An alternative solution for this star gives $T_{\mathrm{eff}}=11600 \mathrm{~K}$ and $\log g=8.0$ (see Sect. 2.2 for more
details).

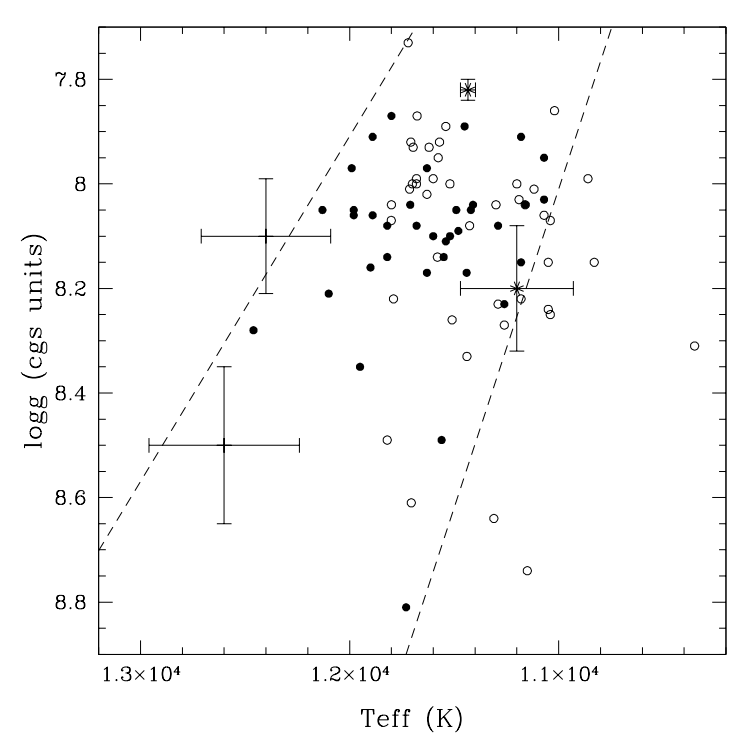

Fig. 6. The observational ZZ Ceti instability strip updated: the two new pulsators are represented by the two asterisks with error bars, whereas the two objects close to the blue edge are HS 1253+1033 and HS 1443+2934 (from left to right), for which no pulsations were found. The filled circles are the known ZZ Ceti stars from Bergeron et al. (2004), the open circles are the new pulsators discovered from the SDSS (Mukadam et al. 2004a; Mullally et al. 2005).

by the SDSS sample alone. Considering that we used the same Koester model atmospheres as used by the SDSS team, the stability against $g$-modes of HS $1253+1033$ and HS $1443+2934$ appears even less surprising.

Acknowledgements. R.S. acknowledges support from the funds COFIN "Astrosismologia" (PI L. Paternò). D.K. and B.V. thank the Deutsche Forschungsgemeinschaft (DFG) for their support (KO738/21-1, KO738/22-1, KO738/23-1). R.N. acknowledges support by a PPARC Advanced Fellowship. D.H. thanks the DFG for grant KO738/10. The authors wish to thank Anjum Mukadam for a very accurate referee report that contributed to improve the quality of this article.

Note added in proof: The discovery of 23 new DAVs was presented recently by B.G. Castanheira during the workshop on "Stellar pulsation and evolution" (Monte Porzio Catone, Roma, 20-24 June 2005).

\section{References}

Araujo-Betancor, S., Gänsicke, B. T., Hagen, H.-J., et al. 2005, A\&A, 430,629

Bergeron, P., Wesemael, F., Fontaine, G., \& Liebert, J. 1990, ApJ, 351, L21

Bergeron, P., Wesemael, F., Lamontagne, R., et al. 1995, ApJ, 449, 258

Bergeron, P., Fontaine, G., Billeres, M., et al. 2004, ApJ, 600, 400

Brickhill, A. J. 1991, MNRAS, 252, 334

Christlieb, N., Wisotzki, L., Reimers, D., et al. 2001, A\&A, 366, 898

Dziembowski, W., \& Koester, D. 1981, A\&A, 97, 16

Dolez, N., \& Vauclair, G., 1981, A\&A, 102, 375

Finley, D. S., Koester, D., \& Basri, G. 1997, AJ, 488, 375

Fontaine, G., Brassard, P., \& Bergeron, P. 2001, PASP, 113, 409

Gautschy, A., Ludwig, H.-G., \& Freytag, B. 1996, A\&A, 311, 493

Goldreich, P., \& Wu, Y. 1999, ApJ, 523, 805

Hagen, H.-J., Groote, D., Engels, D., \& Reimers, D. 1995, A\&AS, 111,195

Homeier, D., \& Koester, D. 2001, 12th European Workshop on White Dwarfs, ed. J. L. Provencal, H. L. Shipman, J. MacDonald, \& S. Goodchild, ASP Conf. Proc., 226, 397

Homeier, D., Koester, D., Hagen, H.-J., et al. 1998, A\&A, 338, 563

Kanaan, A., Nitta, A., Winget, D. E., et al. 2005, A\&A, 432, 219

Koester, D., \& Holberg, J. B. 2001, Proc. of the 12th European Workshop on White Dwarfs, PASP Conf. Ser., 226, 299

Metcalfe, T. S. 2003, ApJ, 587, L43

Mukadam, A., Kepler, S. O., Winget, D. E., et al. 2003, ApJ, 594, 961

Mukadam, A. S., Mullally, F., Nather, R. E., et al. 2004a, ApJ, 607, 982

Mukadam, A. S., Winget, D. E., von Hippel, T., et al. 2004b, ApJ, 612, 1052

Mullally, F., Thompson, S. E., Castanheira, B. G., et al. 2005, Proc. of the 14th European Workshop on White Dwarfs, ed. D. Koester, \& S. Moehler, ASP Conf. Ser., in press [arXiv:astro-ph/0502520]

Napiwotzki, R., Christlieb, N., Drechsel, H., et al. 2003, ESO Messenger, 112, 25

Reif, K., Bagschik, K., de Boer, K. S., et al. 1999, SPIE, 3649, 109

Straniero, O., Domínguez, I., Imbriani, G., \& Piersanti, L. 2003, ApJ, 583,878

Stumpff, P. 1980, A\&AS, 41, 1

Tassoul, M., Fontaine, G., \& Winget, D. E. 1990, ApJS, 72, 335

Warner, B., \& Woudt, P. A. 2005, Proc. of the 14th European Workshop on White Dwarfs, ed. D. Koester, \& S. Moehler, ASP Conf. Ser., in press

Winget, D. E., \& Fontaine, G. 1982, in Pulsations in Classical and Cataclysmic Variable Stars, ed. J. P. Cox, \& J. Hansen (Univ. of Colorado: Boulder), 46

Winget, D. E., Van Horn, H. M., Tassoul, M., et al. 1982, ApJ, 252, L65

Woudt, P. A., \& Warner, B. 2004, MNRAS, 348, 599 